\title{
A Live Environment for Inspection and Refactoring of Software Systems
}

\author{
Sara Fernandes \\ Faculty of Engineering, University of Porto \\ Institute for Systems and Computer Engineering, Technology and Science \\ Porto, Portugal \\ sfcf@fe.up.pt
}

\begin{abstract}
Refactoring helps to improve the design of software systems, making them more readable, maintainable, cleaner, and easy to expand. Most of the tools that already exist on this concept allow developers to select and execute the best refactoring techniques for a particular programming context. However, they aren't interactive and prompt enough, providing a poor programming experience. In this gap, we can introduce and combine the topic of liveness with refactoring methods. Live Refactoring allows to know continuously, while programming, the blocks of code that we should refactor and why they were classified as problematic. Therefore, it shortens the time needed to create high-quality systems, due to early and continuous refactoring feedback, support, and guidance. This paper presents our research project based on a live refactoring environment. This environment is focused on a refactoring tool that aims to explore the concept of Live Refactoring and its main components - recommendation, visualization, and application.
\end{abstract}

\section{CCS CONCEPTS}

- Software and its engineering $\rightarrow$ Real-time systems software; Software maintenance tools; $\bullet$ Human-centered computing $\rightarrow$ Visualization systems and tools.

\section{KEYWORDS}

refactoring, code smells, liveness, code quality, visualization

\section{ACM Reference Format:}

Sara Fernandes. 2021. A Live Environment for Inspection and Refactoring of Software Systems. In Proceedings of the 29th ACM Joint European Software Engineering Conference and Symposium on the Foundations of Software Engineering (ESEC/FSE '21), August 23-28, 2021, Athens, Greece. ACM, New York, NY, USA, 5 pages. https://doi.org/10.1145/3468264.3473100

\section{INTRODUCTION}

Large and complex software systems are hard to understand, maintain and evolve. These could be the most expensive and troubling tasks in the software development cycle because of the possible hidden issues on the way code was developed [Banker et al. 1993]. To

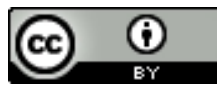

This work is licensed under a Creative Commons Attribution International 4.0 License.

ESEC/FSE '21, August 23-28, 2021, Athens, Greece

(C) 2021 Copyright held by the owner/author(s).

ACM ISBN 978-1-4503-8562-6/21/08.

https://doi.org/10.1145/3468264.3473100 mitigate these problems, we should restructure the code, refactoring it, to create better systems [Fowler 2018].

There are a lot of refactoring tools available for different types of software. However, most of them only focus on showing the code that should be refactored and how it should be done, and not when the refactoring should be executed. Besides, several approaches already try to shorten the feedback loop between each programming action and its results, providing quicker guidance to help developers converging to better programming solutions faster [Martin 2003]. Therefore, Live Refactoring aims to speed-up the refactoring loop, which is composed of three moments: identification, suggestion, and application of refactoring techniques to improve the software under analysis. With it, developers receive immediate and continuous feedback, support, and guidance about the possible refactoring candidates. In this way, they are able to improve their systems while programming them, by being motivated to create better code in earlier development stages.

To research more about this concept, we proposed an approach based on a Live Refactoring Environment. The main goal of our research is to prove if identifying refactoring candidates, recommending them to developers in a visual and non-intrusive way, and applying them automatically, in real-time, would help developers converge to better programming solutions, with quality.

This paper is divided into multiple sections. In Section 2, we provide some background and related work on the main topics of our research project. In Section 3, we detailed our idealized approach for Live Refactoring, by detailing the problem we aim to mitigate, our hypothesis and research questions, main goals, contributions, and expected validation protocol, and our preliminary results. Finally, in Section 4, we draw the main conclusions collected from this research project and outline possible future work.

\section{BACKGROUND \& RELATED WORK}

Our research project is mostly focused on concepts such as Liveness and Refactoring, and other complementary topics like Code Quality Metrics and Software Visualization.

Liveness helps developers to shorten the "edit-compile-link-run" loop by providing possible visual real-time feedback about their software [Aguiar et al. 2019; Fernandes et al. 2020; Tanimoto 2013]. In this topic, we have Circa. Circa is a live language and development environment where developers can interact visually with their systems [Fischer 2013]. Besides, we also have the IntelliJ plugin created by Biegel et al. [Biegel et al. 2015], in which the developers can change their code through a web application, while programming. 
Refactoring is the process that changes the internal structure of software without modifying its external behaviors. This kind of action aims to mitigate code smells. A Code Smell is any surface indicator that can represent a deeper problem on software [Fowler 2018]. With refactoring, developers create cleaner and self-explanatory code. Martin Fowler [Fowler 2018] defined several code smells and related refactorings. Some refactoring tools are focused on them. JDeodorant is and Eclipse and IntelliJ plugin that identifies code smells like Long Method, or God Class, and removes them by applying refactorings like Extract Method or Extract Class [Fokaefs et al. 2011].

Taking into account the strengths of liveness and refactoring, we have the topic of Live Refactoring. Live Refactoring aims at continuously presenting in real-time possible refactoring opportunities that can be applied automatically on the code to improve its readability, adaptability, and maintainability. On this subject, there are already some tools and approaches that provide live refactoring recommendations. Code-Imp is a Java live refactoring tool that detects 14 different refactoring techniques divided into three categories: method-level, field-level, and class-level [Moghadam and Ó Cinnéide 2011; Ó Cinnéide et al. 2012].

To detect refactoring candidates, we can assess several code quality metrics. Code Quality Metrics are based on standard measures of specific software properties that aim to assess their quality[Chidamber and Kemerer 1994; Lanza et al. 2006]. There are a large set of code quality metrics such as the number of lines of code, number of statements, or the effort needed to understand the implemented code [Halstead 1977]. On this topic, we have tools like CodeMetrics or iPlasma. CodeMetrics is a real-time Visual Studio Code plugin that measures the cyclomatic complexity of TypeScript, JavaScript, or Lua code [Tamás 2020]. iPlasma is a tool for Java and $\mathrm{C}++$ systems that uses metrics, like cohesion or coupling, to evaluate the quality of IDEs such as Eclipse or web browers like Mozilla [Marinescu et al. 2005].

Finally, we also need to look for possible approaches that help developers read, change and maintain their software more easily [Caserta and Zendra 2011; Kádár et al. 2016]. In software development, we can represent software through visual methodologies. That is what Software Visualization mainly means. With it, developers are able to understand their systems better, reducing the time needed to achieve good programming solutions [Storey et al. 2000]. SeeSoft is an example of a tool that does exactly that. It is a code-line visualization tool that represents the lines of code through colored pixels [Eick et al. 1992]. CodeCity is also a visualization tool, which allows developers to visualize their software as cities, in real-time [Wettel et al. 2011].

\section{RESEARCH APPROACH}

In this section, we present our research project by describing the problem we aim to solve, our idealized solution, our hypothesis and research questions, main goals, contributions, and the plan that we aim to follow to validate it. We also state our preliminary results.

\subsection{Research Problem}

Every developer wishes to implement good software systems, with high quality. However, this is hard to achieve. Most systems have programming flaws that deteriorate their readability, adaptability, and maintainability [Kádár et al. 2016; Mohan and Greer 2018]. There are several development environments that aim to guide developers towards better software. However, these environments could provide better real-time support, improve awareness on possible problems, and reduce the time needed to achieve cleaner, self-explanatory, and straightforward code [Cassell et al. 2011].

To mitigate this problem, we can inspect the code to find possible defects, guiding programmers about these issues and the ways they should reduce them. However:

How can we search for programming issues and at the same time provide continuous and immediate feedback and support to developers about the quality of their systems, guiding them towards better code?.

Yamashita and Moonen [Yamashita and Moonen 2013] stated that developers want to receive feedback about their software systems as soon as possible. However, inspecting software systems can be tricky and time-consuming since they can have many lines of code and a high complexity degree. We also know that the programming defects that we refer to are also known as code smells, which can be mitigated by applying a refactoring or sequence of refactorings [Opdyke 1992]. But, also finding code smells and refactoring code can be a hard task. Besides, Murphy-Hill et al. [Murphy-Hill and Black 2008, 2010] concluded that most developers refactor their code manually, which can badly increase the time needed to create good software. When they don't refactor their systems manually, they use external and non-real-time tools.

\subsection{Proposed Solution}

Our research project will follow the Engineering Research Method, combined with observational and controlled methods, to validate our approach [Zelkowitz and Wallace 1998]. Therefore, we propose a Live Refactoring Environment capable of reviewing code and measuring specific code quality metrics such as the cyclomatic complexity, the number of statements, or the difficulty of reading code, to detect several code smells and identify possible refactoring opportunities, in real-time. With it, we aim to support and guide developers to create better code with high-quality faster than using conventional refactoring tools. The development of our environment will be divided into distinct phases:

(1) Analysis of Code quality Metrics: In this phase, we expect to measure several code quality metrics to detect specific code smells and identify refactoring opportunities;

(2) Detection of Code Smells: In this phase, we expect to use the assessed metrics to detect specific code smells;

(3) Identification and Suggestion of Refactorings: In this phase, we expect to use the assessed metrics and the detected smells to identify and suggest different refactoring candidates;

(4) Implementation of Liveness: In this phase, we expect to implement live mechanisms to our approach to reduce the refactoring-loop, presenting possible refactorings to developers in real-time.

Furthermore, we also expect to do both laboratorial and robotic experiments to help us validating and refining each phase of our approach. In the end, we hope to do the same but, in this case, to validate each research question and prove our hypothesis. 
3.2.1 Research Statement. The proposed solution is based on the following hypothesis:

"A development environment that continuously inspects source code, detects and visualizes code smells, and recommends refactoring techniques, in real-time, helps software developers to achieve code with more quality and faster."

Taking into account the statement below, we enumerate four different research questions:

RQ1 "Which non-intrusive ways can be used to inform developers, in real-time, of possible code smells in their source code?"

RQ2 "What is the impact of real-time code smell detection on the developer's a wareness of code quality?"

RQ3 "What is the impact of a real-time refactoring environment on the quality of source code?"

RQ4 "What is the impact of a live refactoring environment in the time needed to reach good programming solutions?"

These questions aim to validate different aspects of our solution, such as if it creates awareness to developers towards possible code smells, improves the quality of the code, or reduces the time needed to create good software.

3.2.2 Main Goals. As it was said, this research project aims to improve and speed-up the feedback, support, and guidance provided to developers about the quality of their software, to help them achieve better code, by:

(1) Augmenting the awareness about possible code quality flaws;

(2) Helping programmers making better programming decisions, by suggesting possible refactorings to be applied in a specific context;

(3) Speeding-up the convergence to a high-quality programming solution.

3.2.3 Expected Contributions. The expected results of our research project are divided into the following components:

State-of-the-Art. To know which are the advances on the most relevant subjects of our project, we conducted a literature review (Section 2) on code smells, refactoring, code quality metrics, liveness, and software visualization;

Live Refactoring Environment. We proposed a live refactoring environment to mitigate the problem described in Section 3.1. This environment should be capable of reviewing code and measuring specific code quality metrics such as the cyclomatic complexity or the maintainability degree, to detect different code smells such as Large Class, Long Method, or Feature Envy, and to identify refactoring opportunities like Extract Class, Extract Method, or Move Method, in real-time;

Empirical Experiments. After we finish developing our tool, we also plan to execute empirical experiments to help us validate it. We aim to use academic and industrial case studies with real and robotic participants to verify if our solution is effective and if we can answer our research questions and prove our hypothesis.

3.2.4 Expected Validation Methodology. To validate our research project, we aim to incrementally perform some tests over the different parts of our approach to verify if we developed it correctly. In the end, we also aim to execute an empirical experiment, but in this case, including all aspects of our solution, We expect to use academic and industrial case studies, in which programmers or programming students will have to perform different refactoring tasks. We hope to have at least 50 participants. These participants won't need to fulfill any particular requirement. We believe that they only need to know Java, IntelliJ, and the refactoring process. In the last experiment, the participants will be divided into two distinct groups, one that will use the developed tool and other that won't use any auxiliary tool to perform the refactoring tasks.

With the different tasks, we aim to collect enough data related to each of our research questions. We expect to measure the usability of our tool using a System Usability Scale (SUS), and by counting the number of times, each participant used the tool. We also want to measure the impact of our approach on raising awareness on code quality, by measuring how many times the participants accepted the refactorings suggested by the tool. To measure code quality, to help us verify if the refactorings suggested and applied by the tool guided the participants to better code, we will compare the metrics before and after each refactoring. Finally, to assess the time performance of our tool, we expect to compare the time needed to finish each task with and without our tool.

3.2.5 Preliminary Results. So far, we were able to conduct a large literature review mainly on Liveness, Refactoring, Code Quality Metrics, and Software Visualization.

Besides, we started implementing our Live Refactoring Environment. Currently, it only works for IntelliJ IDE, for Java software systems. To summarize, this environment measures several code quality metrics and other software aspects, in real-time, to identify refactoring opportunities on the source code. Then, each refactoring is suggested to developers, and if they accept them, they are applied automatically on the code.

As we said, we measured several metrics such as the number of lines of code, number of properties of a class, number of methods, cyclomatic complexity, Halstead metrics, lack of cohesion, conceptual structural similarity, or dependency between methods. Most of the metrics were assessed using the Program Structure Interface (PSI) created by the IDE, which can be accessed through its API.

Then, our tool uses some of these metrics as heuristics to create an extract-related refactoring recommendation system. On this first development phase, we chose to only focus on detecting the Extract Method, Extract Class, and Extract Variable refactorings, since they can solve frequent problems like long methods classes, duplicated code, or complex statements [Fowler 2018].

To detect Extract Method opportunities, we used the cyclomatic complexity, the number of statements, the number of lines of code, and the lack of cohesion. If all of these metrics have values bigger than the thresholds defined by us and that can be established by the users through a configuration menu, and if the nodes classified as possible refactoring candidates are consecutive, the system saves that code as an extract method candidate.

To find Extract Class candidates, we measured three MxM matrices (with $\mathrm{M}$ being the number of methods in the target class). The matrices represent the Conceptual Similarity between Methods (CSM), Call-based Dependence between Methods (CDM), and Structural Similarity between Methods (SSM). After calculating all three matrices, we measured a final weight matrix where the values 
on the first row represented if the methods should be extracted to a new class or not. If the values are on the interval ]0.15; 0.30 ], the respective methods are pointed out as extract candidates.

We also created a mechanism to find Extract Variable refactoring opportunities. In this case, we analyze each call expression of a specific code file, and we verify if that call is related or not with a void returning method. To do so, we checked each method present on the project being analyzed and also the return type of that call expression. If it isn't related to a void returning method, and if its length is bigger than the respective threshold, the expression is saved as a possible extract variable candidate.

As already mentioned, we have different thresholds for each type of detection that the users can configure. We decided to do it this way, because we found hard to define a final threshold that should be used on every occasion, in every programming context.

After detecting the extract candidates, our system sorts them inside each category. For extract methods, it uses the number of statements or the lack of cohesion. For extract classes, it uses the number of methods to be extracted. And for extract variables, it compares the length of the expressions to be extracted.

Then, we sort all the candidates by severity where it takes into account the number of candidates detected, and that should be distributed by a system of colors created by us that contains ten shades in a range of green and red, where green represents code that doesn't need to be refactored, and red represents code that needs to be extracted. Through the color representation, painted on the left side of the text editor, users can choose which code they want to refactor based on the suggestions (Figure 1). If users click on one of the colors, they will have access to the different refactoring candidates associated with that node or with that color. If they accept the suggestions, the code extraction is made automatically using the built-in commands for method, class, or variable extraction provided by the IntelliJ API.

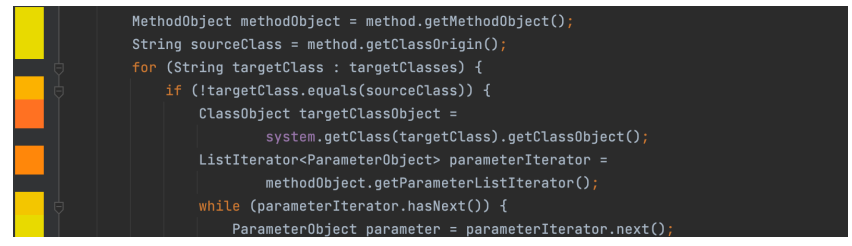

Figure 1: Visual representation of refactoring candidates.

As the last step, we included some live mechanisms that helped make our approach as quick and continuous as possible. To do so, we used a listener that triggers an event when we change the code that is being displayed on the text editor or when we change the document focused by the editor. Every time the event is triggered, we save the old metrics, and we measure the new ones, and we start the process to find new refactoring candidates.

We weren't able yet to conduct some empirical experiments with different participants. However, we compare some data obtained while creating our tool on the code quality metrics before and after applying a refactoring. The results obtained so far, even not being enough to prove our hypothesis, have shown us that we are on the right track to having a live refactoring environment capable of improving the quality of the code, while programming.

\section{CONCLUSIONS}

Software systems can be complex, hard to understand, adapt, and maintain. To address this problem, developers usually inspect their code to find possible programming flaws like code smells. To mitigate these smells, they need to refactor the code. However, identifying possible refactoring candidates isn't an easy task when the software has low readability. To mitigate this issue, we consider that a refactoring suggestion environment would reduce the effort needed to restructure code to improve its quality, raising its capability to be understood, changed, and maintained. We also think that by including a higher level of liveness in this topic, developers would be able to improve their systems quickly.

We believe that a Live Refactoring Environment that automatically and continuously inspects and refactors code will positively influence the feedback, support, and guidance loop between developers and their software. Thus, this research project aims to assess code quality metrics to detect blocks of code that should be refactored, and that will be visually identified in the text editor, in real-time, to improve the awareness of developers of the flaws implemented on their systems. Besides, with quicker feedback, we expect to reduce the time needed to create high-quality programming solutions.

Our preliminary results show exactly that. We immediate and continuous visual information about code refactoring opportunities, we were able to improve the quality of the code by mitigating preexisting code smells. Also, they have shown that a live refactoring environment helps to achieve better solutions, with better quality, faster. Therefore, although not firm yet, these results are motivating enough to continue researching novel ways to add more liveness to the overall refactoring process and activities and include even more refactoring methods to our approach.

\subsection{Future Work}

As future work, we expect to include in our approach the detection of other code smells such as Shotgun Surgery, Duplicate Code, or Lazy Class. Then, to solve this problems we also aim to introduce new refactoring opportunities like Move Method, Substitute Algorithm, or Inline Class [Fowler 2018]. We will need to measure more code quality metrics and use different algorithms and techniques to detect new possibilities of refactoring on the code. Besides, we also want to use smart algorithms to calculate the best sequence of refactorings to be applied in a specific programming context.

After developing this tool, we aim to execute some empirical experiments to evaluate our approach and assess the development experience provide by the environment. We expect to use academic and industrial case studies, where the participants will have different tasks to fulfill. Furthermore, we aim to analyze the data collected using hypothesis tests. In this way, we believe that we will be able to conclude if our experiment helps developers improve their attitude towards better software systems, with better code, quickly.

\section{ACKNOWLEDGMENTS}

This work was funded by the Portuguese Foundation for Science and Technology (FCT), under the research grant 2020.05161.BD, and it was hosted by the Faculty of Engineering, University of Porto (FEUP), and the Institute for Systems and Computer Engineering, Technology and Science (INESC-TEC). 


\section{REFERENCES}

Ademar Aguiar, André Restivo, Filipe Figueiredo Correia, Hugo Sereno Ferreira, and João Pedro Dias. 2019. Live Software Development: Tightening the Feedback Loops. In Proceedings of the Conference Companion of the 3rd International Conference on Art, Science, and Engineering of Programming (Genova, Italy) (Programming '19). Association for Computing Machinery, New York, NY, USA, Article 22, 6 pages. https://doi.org/10.1145/3328433.3328456

Rajiv D Banker, Srikant M Datar, Chris F Kemerer, and Dani Zweig. 1993. Software complexity and maintenance costs. Commun. ACM 36, 11 (1993), 81-95.

Benjamin Biegel, Benedikt Lesch, and Stephan Diehl. 2015. Live Object Exploration Observing and Manipulating Behavior and State of Java Objects. In Proceedings of the 2015 IEEE International Conference on Software Maintenance and Evolution (ICSME) (ICSME '15). IEEE Computer Society, USA, 581-585. https://doi.org/10. 1109/ICSM.2015.7332518

Pierre Caserta and Olivier Zendra. 2011. Visualization of the Static Aspects of Software: A Survey. IEEE Transactions on Visualization and Computer Graphics 17, 7 (jul 2011), 913-933. https://doi.org/10.1109/TVCG.2010.110

Keith Cassell, Craig Anslow, Lindsay Groves, and Peter Andreae. 2011. Visualizing the refactoring of classes via clustering. Conferences in Research and Practice in Information Technology Series 113, Acsc (2011), 63-72.

S.R. Chidamber and C.F. Kemerer. 1994. A metrics suite for object oriented design. IEEE Transactions on Software Engineering 20, 6 (jun 1994), 476-493. https://doi. org/10.1109/32.295895

S. C. Eick, J. L. Steffen, and E. E. Sumner. 1992. Seesoft-a tool for visualizing line oriented software statistics. IEEE Transactions on Software Engineering 18, 11 (1992), 957-968.

Sara Fernandes, André Restivo, Hugo Sereno Ferreira, and Ademar Aguiar. 2020. Helping Software Developers through Live Software Metrics Visualization. In Conference Companion of the 4th International Conference on Art, Science, and Engineering of Programming (Porto, Portugal) ('20). Association for Computing Machinery, New York, NY, USA, 209-210. https://doi.org/10.1145/3397537.3397539

Andrew Fischer. 2013. Introducing circa: A Dataflow-Based Language for Live Coding. In Proceedings of the 1st International Workshop on Live Programming (LIVE '13). IEEE Press, San Francisco, California, 5-8.

Marios Fokaefs, Nikolaos Tsantalis, Eleni Stroulia, and Alexander Chatzigeorgiou. 2011. JDeodorant: Identification and Application of Extract Class Refactorings. In Proceedings of the 33rd International Conference on Software Engineering (Waikiki, Honolulu, HI, USA) (ICSE '11). Association for Computing Machinery, New York, NY, USA, 1037-1039. https://doi.org/10.1145/1985793.1985989

Martin Fowler. 2018. Refactoring: Improving the Design of Existing Code (2nd Edition). Addison-Wesley, USA.

Maurice H. Halstead. 1977. Elements of Software Science (Operating and Programming Systems Series). Elsevier Science Inc., USA.

István Kádár, Péter Hegedundefineds, Rudolf Ferenc, and Tibor Gyimóthy. 2016. A Manually Validated Code Refactoring Dataset and Its Assessment Regarding Software Maintainability. In Proceedings of the The 12th International Conference on Predictive Models and Data Analytics in Software Engineering (Ciudad Real, Spain) (PROMISE 2016). Association for Computing Machinery, New York, NY, USA, Article 10, 4 pages. https://doi.org/10.1145/2972958.2972962

Michele Lanza, Radu Marinescu, and Stéphane Ducasse. 2006. Object-Oriented Metrics in Practice. Springer Berlin Heidelberg, Berlin, Heidelberg. https://doi.org/10.1007/3-
540-39538-5

Cristina Marinescu, Radu Marinescu, Petru Mihancea, Daniel Ratiu, and Richard Wettel. 2005. iPlasma:An Integrated Platform for Quality Assessment of ObjectOriented Design. In Proceedings of the 21st IEEE International Conference on Software Maintenance ICSM 2005. ICSM (Industrial and Tool Volume), Budapest, Hungary, $77-80$.

Robert Cecil Martin. 2003. Agile Software Development: Principles, Patterns, and Practices. Prentice Hall PTR, USA

Iman Hemati Moghadam and Mel Ó Cinnéide. 2011. Code-Imp: A Tool for Automated Search-Based Refactoring. In Proceedings of the 4th Workshop on Refactoring Tools (Waikiki, Honolulu, HI, USA) (WRT '11). Association for Computing Machinery, New York, NY, USA, 41-44. https://doi.org/10.1145/1984732.1984742

Michael Mohan and Des Greer. 2018. A survey of search-based refactoring for software maintenance. Journal of Software Engineering Research and Development 6, 1 (07 Feb 2018), 3. https://doi.org/10.1186/s40411-018-0046-4

Emerson Murphy-Hill and Andrew P Black. 2008. Refactoring tools: Fitness for purpose. IEEE software 25, 5 (2008), 38-44.

Emerson Murphy-Hill and Andrew P. Black. 2010. An Interactive Ambient Visualization for Code Smells. In Proceedings of the 5th International Symposium on Software Visualization (Salt Lake City, Utah, USA) (SOFTVIS '10). Association for Computing Machinery, New York, NY, USA, 5-14. https://doi.org/10.1145/1879211.1879216

Mel Ó Cinnéide, Laurence Tratt, Mark Harman, Steve Counsell, and Iman Hemati Moghadam. 2012. Experimental Assessment of Software Metrics Using Automated Refactoring. In Proceedings of the ACM-IEEE International Symposium on Empirical Software Engineering and Measurement (Lund, Sweden) (ESEM '12). Association for Computing Machinery, New York, NY, USA, 49-58. https: 1/doi.org/10.1145/2372251.2372260

William F. Opdyke. 1992. Refactoring: A Program Restructuring Aid in Designing Object-Oriented Application Frameworks. Ph.D. Dissertation. University of Illinois at Urbana-Champaign.

M.-A.D. Storey, K. Wong, and H.A. Müller. 2000. How do program understanding tools affect how programmers understand programs? Science of Computer Programming 36, 2-3 (mar 2000), 183-207. https://doi.org/10.1016/S0167-6423(99)00036-2

Kiss Tamás. 2020. CodeMetrics. https://marketplace.visualstudio.com/items? itemName=kisstkondoros.vscode- codemetrics.

Steven L. Tanimoto. 2013. A Perspective on the Evolution of Live Programming. In Proceedings of the 1st International Workshop on Live Programming (San Francisco, California) (LIVE '13). IEEE Press, Piscataway, NJ, USA, 31-34. http://dl.acm.org/ citation.cfm?id=2662726.2662735

Richard Wettel, Michele Lanza, and Romain Robbes. 2011. Software Systems as Cities: A Controlled Experiment. In Proceedings of the 33rd International Conference on Software Engineering (Waikiki, Honolulu, HI, USA) (ICSE '11). Association for Computing Machinery, New York, NY, USA, 551-560. https://doi.org/10.1145/ 1985793.1985868

Aiko Yamashita and Leon Moonen. 2013. Do developers care about code smells? An exploratory survey. In 2013 20th Working Conference on Reverse Engineering (WCRE). IEEE Press, San Francisco, California, 242-251. https://doi.org/10.1109/WCRE.2013. 6671299

M. V. Zelkowitz and D. R. Wallace. 1998. Experimental models for validating technology. Computer 31, 5 (1998), 23-31. 